\title{
Director movement and potential function of surface stabilized ferroelectric liquid crystal cells by electrooptical investigations
}

\author{
FRANK GIEßELMANN and PETER ZUGENMAIER \\ Institut für Physikalische Chemie der Technischen Universität Clausthal, \\ Arnold-Sommerfeld-Strasse 4, D-3392 Clausthal-Zellerfeld, F.R. Germany
}

(Received 8 November 1989; accepted 5 March 1990)

\begin{abstract}
Electrooptical investigations on a ferroelectric liquid crystal (DOBAMBC) were carried out on a well aligned sample in a $2 \mu \mathrm{m}$ cell. On the basis of these measurements the complete average director movement during a switching process caused by an electric field can be calculated and will be discussed. The thermodynamic potentials at the $S_{A} \cdot-S_{C}$. phase transition were determined from the experimental results with a modified Landau theory. They serve as a basis for a discussion of the phase transition and the influence of the surface orientation. Some preliminary results were obtained concerning the electrooptical behaviour of the $\mathrm{S}_{\mathrm{H}}$. phase.
\end{abstract}

\section{Introduction}

Ferroelectric liquid crystals have developed to an important field initiated by the work of Meyer et al. in 1975 [1]. This material is not only of great interest for scientific research but also for applications as well as, for example, in fast bistable electrooptical devices.

Electrooptical investigations of oriented ferroelectric samples in thin cells are frequently used for a characterization of the material, especially for the measurement of switching times. Furthermore, a detailed analysis of electrooptical experiments may provide quantitative information of director movement, potential functions and surface effects. The experimental results are accessible by the application of a modified Landau theory.

\section{Theoretical}

The starting point for a thermodynamical description of ferroelectric smectic phases is very often represented by the Landau theory in which the polarization or the director tilt angle is introduced as a characteristic order parameter [2].

The director $\hat{n}$ can be described as a function of the azimuthal and polar angles $\varphi, \vartheta$ with respect to the smectic layer normal $\hat{z}$, if a uniform director field is assumed with planar smectic layers perpendicular to the glass plates of a thin cell (figure 1)

$$
\hat{n}=\left(\begin{array}{c}
\sin \vartheta \\
\cos \vartheta \sin \varphi \\
\cos \vartheta \cos \varphi
\end{array}\right) \quad \hat{z}=\left(\begin{array}{l}
0 \\
0 \\
1
\end{array}\right) \text {. }
$$




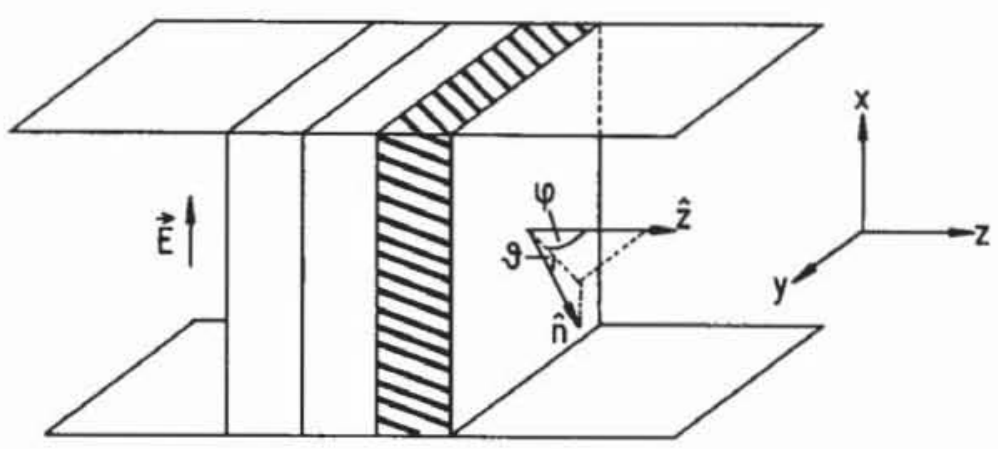

Figure 1. Geometry of an idealized surface stabilized cell with an uniform orientation of smectic layers perpendicular to the glass plates. (E, electric field; $\hat{z}$, smectic layer normal; $\hat{n}$, director; $\varphi$, azimuthal director tilt angle; $\vartheta$, polar director tilt angle.)

The resulting polarization $\mathbf{P}$ is then expressed by

$$
\mathbf{P}(\varphi, \vartheta)=P_{0}[\hat{n} \times \hat{z}]=P_{0}\left(\begin{array}{c}
\cos \vartheta \sin \varphi \\
\sin \vartheta \\
0
\end{array}\right) \text {, }
$$

with $P_{0}$ the maximal size of the vector $\mathbf{P}$ when $\hat{n}$ perpendicular to $\hat{z}$.

The relationship between the thermodynamic potential $g$ and the polarization, omitting other terms, is given at isothermal conditions by

$$
d g=\mathbf{E} d \mathbf{P}=E_{x} d P_{x}+E_{y} \mathrm{~d} P_{y}+E_{z} d P_{z},
$$

with $d P_{z}=0$ from equation (2). Assuming that the internal field is negligibly small as compared with the external applied field $E\left(E_{y} \ll E_{x} \approx E\right)$ follows

$$
d g=E d P_{x} .
$$

Selecting the azimuthal director tilt angle $\varphi$ as a parameter for further calculations, $d P_{x}(\varphi)$ can be expressed with the use of equation (2)

$$
\begin{aligned}
d P_{x} & =\left(\frac{\partial P_{x}}{\partial \varphi}\right)_{s} d \varphi+\left(\frac{\partial P_{x}}{\partial \vartheta}\right)_{\varphi} d \vartheta \\
& =\left[\left(\frac{\partial P_{x}}{\partial \varphi}\right)_{s}+\left(\frac{\partial P_{x}}{\partial \vartheta}\right)_{\varphi}\left(\frac{\partial \vartheta}{\partial \varphi}\right)_{P_{x}}\right] d \varphi \\
& =P_{0}\left[\cos \vartheta \cos \varphi-\sin \vartheta \sin \varphi\left(\frac{\partial \vartheta}{\partial \varphi}\right)_{P_{x}}\right] d \varphi .
\end{aligned}
$$

With equation ( $\left.3^{\prime}\right)$ the following expression for $E$ is obtained:

$$
E=\frac{1}{P_{0}}\left(\cos \varphi \cos \varphi-\sin \vartheta \sin \varphi\left(\frac{\partial \vartheta}{\partial \varphi}\right)_{P_{x}}\right)^{-1} \frac{d g}{d \varphi} .
$$

The direction of $\hat{n}$ and $-\hat{n}$ are equivalent for liquid crystals and evoke a periodicity of $g(\varphi)$ with $\pi$. It is also an even function in the original Landau theory. Since an additional influence of the asymmetric orientational surface has to be assumed, caused by the alignment procedure, odd terms in $g$ have also to be considered. A $\pi$ 


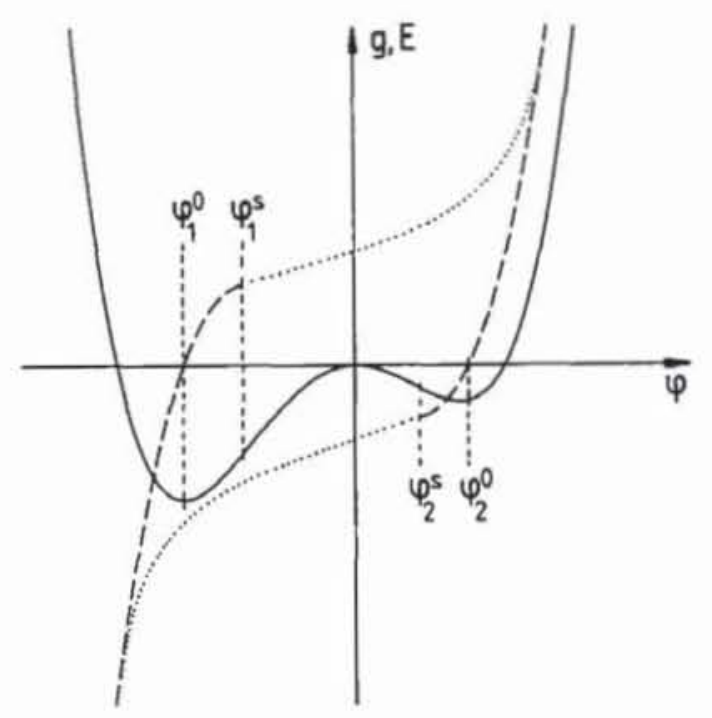

Figure 2. Schematic representation of relations between an asymmetrically distorted potential function and the connected hysteresis curves. (Solid line, potential function; dashed line, thermodynamically controlled hysteresis; dotted line, kinetically controlled hysteresis.)

periodic Fourier expansion of $g(\varphi)$ fulfils these conditions

$$
g(\varphi)=\sum_{v=0}^{\infty}\left[a_{v} \sin (2 v \varphi)+b_{v} \cos (2 v \varphi)\right]
$$

Introduced into equation (5)

$$
\begin{aligned}
E(\varphi)= & \frac{2}{P_{0}}\left(\cos \vartheta \cos \varphi-\sin \vartheta \sin \varphi\left(\frac{\partial \vartheta}{\partial \varphi}\right)_{P_{r}}\right)^{-1} \\
& \times \sum_{v=1}^{\infty}\left[a_{v} v \cos (2 v \varphi)-b_{v} v \sin (2 v \varphi)\right] .
\end{aligned}
$$

The $\varphi, E$ relationship is experimentally available and schematically represented in figure 2. It may be derived from a potential function $g$, also shown in figure 2. A typical ferroelectric potential exhibits two comparable minima separated by a maximum. In figure 2 this equivalence is distorted by an orientational surface influence. The analytical correlation between $g$ and $E$ is given by equation (5) and leads, with the Fourier series of equation (6), to equation (7). According to equation (5), $E(\varphi)$ is essentially proportional to the first derivative of the potential curve excluding the thermodynamically unstable region for the material and assuming small values in $\vartheta$ and $\varphi$ for which the front factor becomes a constant. $E(\varphi)$ passes through zero at $\varphi_{i}^{0}$ and $\varphi_{2}^{0}$, the minima for the potential $g$. At the inflexion points $\varphi_{1}^{s}$ and $\varphi_{2}^{s}$ of the potential, the system becomes unstable $\left(\partial^{2} g / \partial \varphi^{2}<0\right)$ and the $E$ curves leave the thermodynamically controlled pathway with dielectric losses to find their kinetically controlled paths to the stable regions (dotted lines in figure 2).

Therefore, if it is possible to measure $\varphi$ and $\vartheta$ as a function of the applied field $E$ and to fit the thermodynamically allowed region of the $\varphi, E(\varphi)$ hysteresis curve, the potential function $g$ can be deducted. Equation (6) and (7) may serve as a suitable approach for an evaluation of the experimental results. 


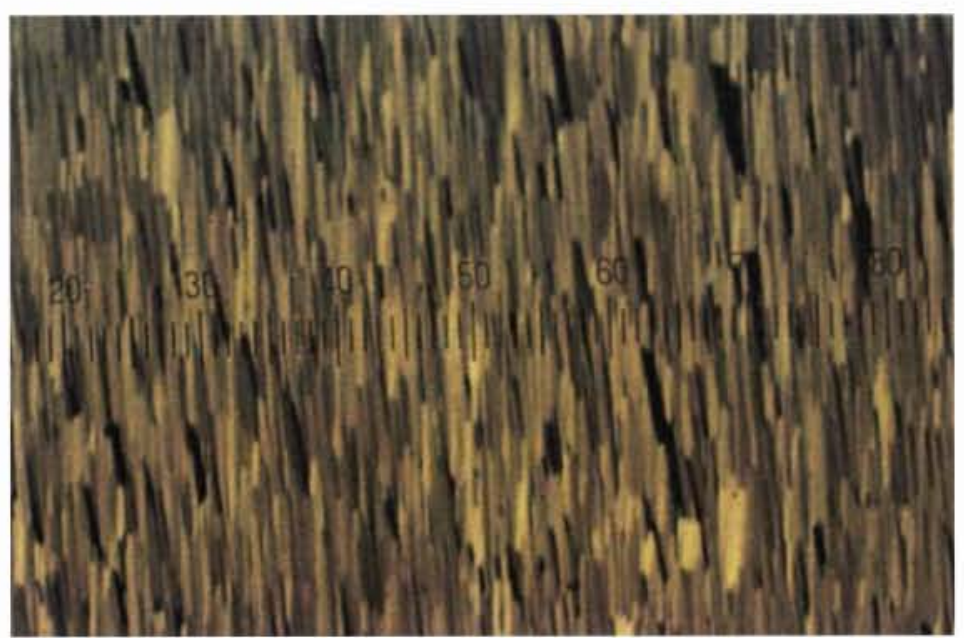

Figure 3.

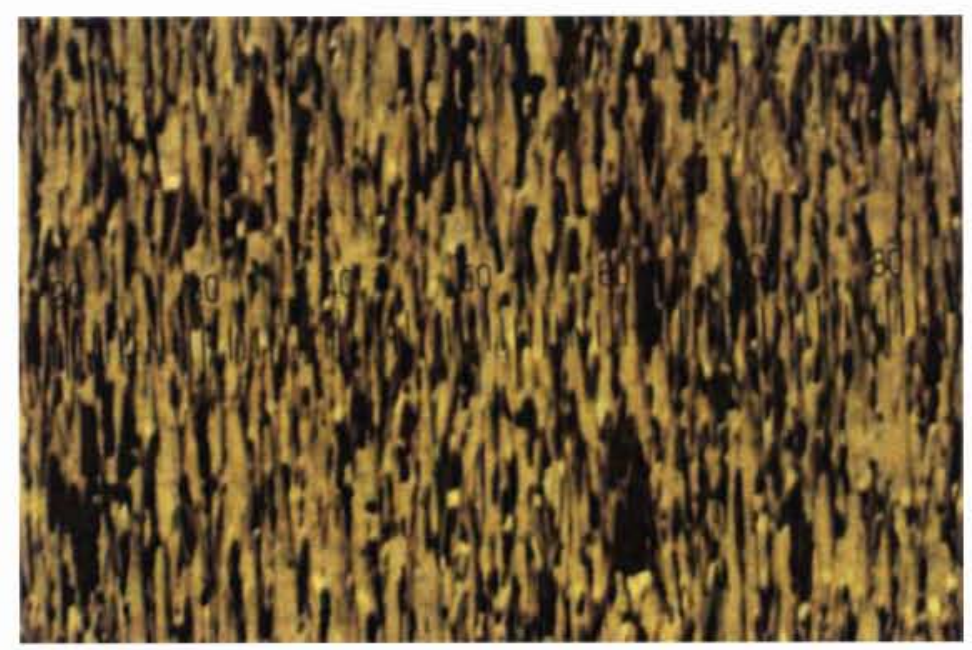

Figure 4.

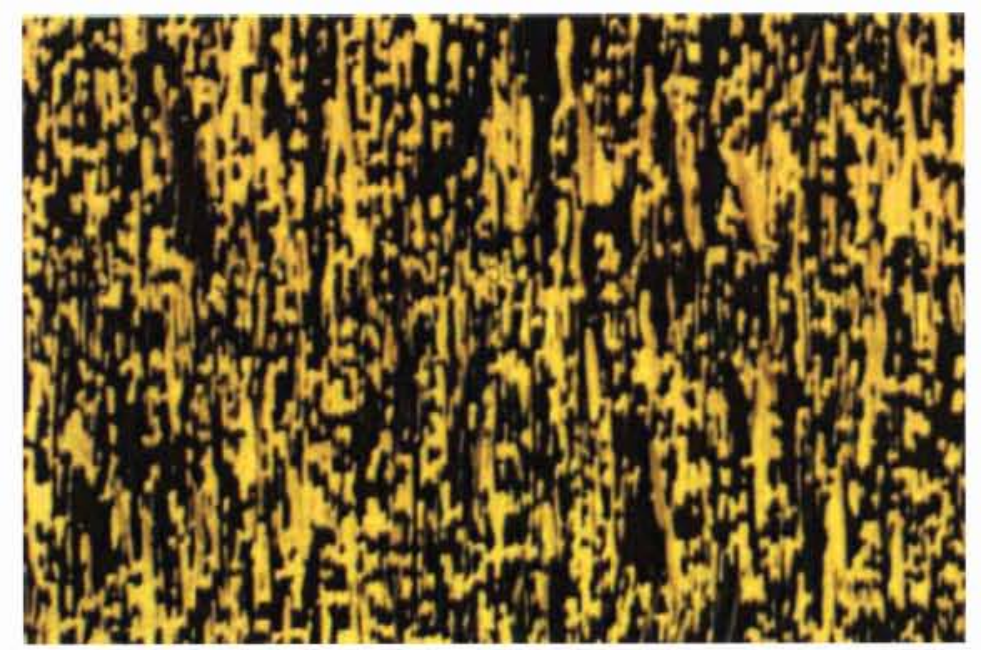

Figure 5. 


\section{Experimental}

\subsection{Material and cell preparation}

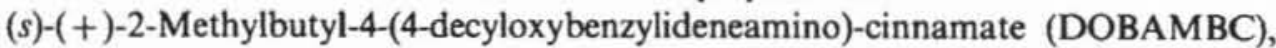
purity 97 per cent, supplied by Aldrich Chem. Co., was used as material in this investigation with the phase sequence [3]

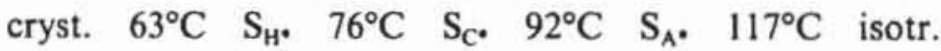

A commercially available Ito cell, thickness $2 \mu \mathrm{m}$, with an orienting polyimide surface served for all measurements. Figures 3-5 demonstrate the good, homogeneous alignment achieved for this material. The morphology of the $\mathrm{S}_{\mathrm{A}^{*}}$. phase is carried over to the $\mathrm{S}_{\mathrm{C}}$, and $\mathrm{S}_{\mathrm{H}}$. phases. The actual sections of the cell investigated in this study are depicted at different temperatures at cooling. The phase transition temperatures observed in this $2 \mu \mathrm{m}$ thick cell by polarization microscopy differ considerably from the ones of [3].

\section{isotr. $\quad 115-110^{\circ} \mathrm{C} \quad \mathrm{S}_{\mathrm{A}^{*}} \cdot 83^{\circ} \mathrm{C} \quad \mathrm{S}_{\mathrm{C}^{*}} \quad 55^{\circ} \mathrm{C} \quad \mathrm{S}_{\mathrm{H}^{*}}$ cryst.}

The isotropic- $\mathrm{S}_{\mathrm{A}}$. transition occurs very indistinctly between 115 and $110^{\circ} \mathrm{C}$. Figure 3 represents the completely formed $\mathrm{S}_{\mathrm{A}}$. phase at $90^{\circ} \mathrm{C}$. The $\mathrm{S}_{\mathrm{A}^{*}}-\mathrm{S}_{\mathrm{C}^{*}}$ transition follows and becomes visible by a separation of up and down domains in the texture. First pre-transitional textural changes can be observed at $85^{\circ} \mathrm{C}$, however, the majority of the structural changes occurs at $83^{\circ} \mathrm{C}$. The texture of the $\mathrm{S}_{\mathrm{C}}$. phase is shown in figure 4 at $80^{\circ} \mathrm{C}$. The absence of parallel domain stripes and zig-zag lines in the homogeneous texture is indicative that neither a helicoidal nor a chevron superstructure is present. A uniform alignment of planar smectic layers has to be assumed, placed more or less perpendicular to the glass plates and schematically drawn in figure 1 (bookshelf geometry).

The next phase transition is not visible before $55^{\circ} \mathrm{C}$, noticeable as a relatively sharp characteristic change in shape and colour of the domains. Figure 5 represents the phase, proposed by Keller et al. [3] as $\mathrm{S}_{\mathrm{H}}$. phase, at $54^{\circ} \mathrm{C}$. At this temperature the phase can be observed for several hours before crystallization takes place.

\subsection{Experimental setup}

A schematic drawing of the setup used in the electrooptical experiments is represented in figure 6 . The cell with the liquid-crystalline material is inserted into a hot stage (Mettler FP 52) and wired to a wideband amplifier (Krohn-Hite Mod. 7500, DC to I MHz, $75 \mathrm{~W}$ ). The inlet of the amplifier is connected to a pulse function generator (Hewlett-Packard $8116 \mathrm{~A}, 50 \mathrm{MHz}$ ). The hot stage with the cell is placed in a polarizing microscope (Olympus $\mathrm{BH} 2$ ), equipped with a photo tube on which a photomultiplier (RCA 1 P 28 with PR 1402 RF tube housing) is fastened. The photo current is

Figure 3. Texture of the well aligned homogeneously oriented $\mathrm{S}_{\mathrm{A}^{*}}$. phase in a $2 \mu \mathrm{m}$ cell observed between crossed polars in the microscope at $84^{\circ} \mathrm{C}$. Actually investigated section; 10 scale bars $\hat{=} 71 \mu \mathrm{m}$.

Figure 4. Texture of the $\mathrm{S}_{\mathrm{C}}$. phase in a $2 \mu \mathrm{m}$ cell, observed between crossed polars in the microscope at $80^{\circ} \mathrm{C}$, showing a diffuse separation of up and down domains. Actually investigated section; same magnification as in figure 3.

Figure 5. Texture of the $\mathrm{S}_{\mathrm{H}}$. phase in a $2 \mu \mathrm{m}$ cell, observed between crossed polars in the microscope at $54^{\circ} \mathrm{C}$ showing sharp separation of up and down domains. Actually investigated section; same magnification as in figure 3. 


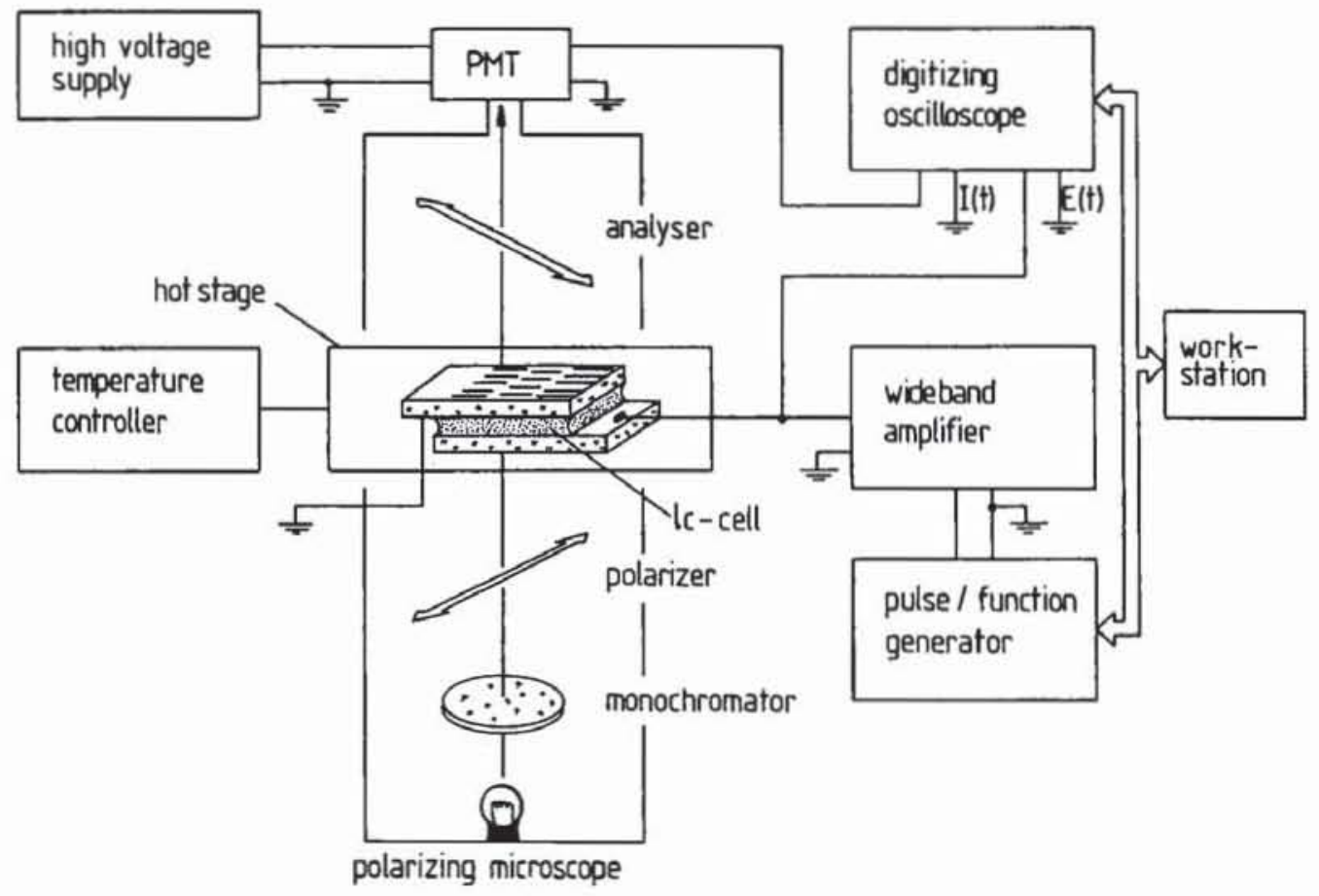

Figure 6. Experimental setup.

measured through a load resistance by one channel of a two-channel digitizing oscilloscope (Hewlett-Packard $54200 \mathrm{~A}$ ). With the other channel the applied voltage to the cell after amplification is recorded over a 10:1 probe divider. The light for the microscopic observations is monochromatized by an interference filter (LOT, $633 \pm 5 \mathrm{~nm}$ ) before passing through the polarization filter. The transmission of the cell in monochrome light is then recorded as a function of the applied electric field.

The transmission scale is set to 0 per cent for the photo current at crossed polarizers without the cell and 100 per cent at parallel polarizers. Reproducible results are obtained if the 100 per cent defined current is measured with reduced light intensity of about 30-40 per cent of the maximum photo current of the device. A long warm-up time is necessary and the 100 per cent current level has been tested to reach a constant value over a longer period of time.

The oscilloscope and the function generator are connected by an HPIB bus to a PS/2 workstation (Hewlett-Packard Vectra RS 16) for data evaluation and remote control.

\subsection{Determination of azimuthal and polar tilt angles of the director}

The transmission of a liquid crystal cell of thickness $d$ between crossed polarizers is given by equation (8) in monochrome light $[4,5]$, wavelength $\lambda$, with $\varphi_{0}$ the angle between $\mathrm{E}$, the electric vector of the polarized light, and the smectic layer normal

$$
T=T_{0} \sin ^{2}\left[2\left(\varphi+\varphi_{0}\right)\right] \sin ^{2}(\pi d \Delta n / \lambda) .
$$

The birefringence $\Delta n$ is only a function of the polar angle $\vartheta$, if the optically biaxial $\mathrm{S}_{\mathrm{C}}$. phase is approximated by an uniaxial system $\left(n_{1} \approx n_{2}=n_{\mathrm{o}}, n_{3}=n_{\mathrm{e}}\right)$ [5].

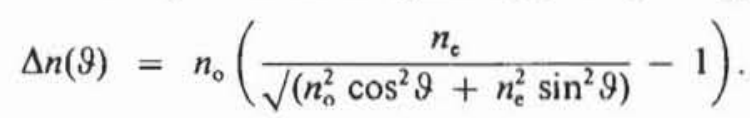


The angles $\varphi$ and $\vartheta$ are determined in two steps. Firstly, the transmission $T_{1}(\varphi)$ is measured as described by equation (8). Secondly, the polarizer and analyser are rotated by $45^{\circ}, \varphi_{0}$ has then to be replaced by $\varphi_{0}+45^{\circ}$ in equation (8), resulting in $T_{2}(\varphi)$ for the transmission:

$$
T_{2}=T_{0} \cos ^{2}\left[2\left(\varphi+\varphi_{0}\right)\right] \sin ^{2}(\pi d \Delta n / \lambda) .
$$

$\varphi$ and $\Delta n(\vartheta)$ are deduced from equation (8) and $\left(8^{\prime}\right)$

$$
\begin{aligned}
\varphi & =\frac{1}{2} \arctan \sqrt{ }\left(\frac{T_{1}}{T_{2}}\right)-\varphi_{0}, \\
\Delta n & =\frac{\lambda}{\pi d} \arcsin \sqrt{ }\left(\frac{T_{1}+T_{2}}{T_{0}}\right) .
\end{aligned}
$$

A numerical solution of equation (9) with known refractive indices $n_{\mathrm{o}}$ and $n_{\mathrm{e}}$ leads to a determination of $\vartheta$. It should be noted, that this procedure is only valid for relative thin cells for which a unique inverse function exists, for which the phase of $\sin (\pi d \Delta n / \lambda)$ lies below $\pi / 2$. It was found that the resulting $\vartheta$ values are not very sensitive to changes of the refractive indices which have to be only known approximately to $2 \times 10^{-2}$.

\subsection{Determination of the potential function}

The potential functions are available by an analysis of the $E, \varphi$ hysteresis curves. Equation (7) will be fitted with a limited number of expansion terms by a least squares method in the two thermodynamically controlled parts which are joined by a straight line in a preliminary approximation. The best fits are obtained with 6 or 8 parameters corresponding $v=3$ or 4 in equation (7). Larger numbers of parameters lead to dependencies of the fitted parameters from each other, resulting in singular coefficient matrices by solving the system of equations. Lower numbers of parameters diminish the quality of the fit expressed as the sum of error squares.

The potential curves are determined according to equation (6), if the expansion coefficients $a_{v}$ and $b_{v}$ are introduced from the best least squares fits. The orientational surface influence is neglected when all $a_{v}$ are set to zero since an even Landau potential function is then expected.

\section{Results and discussion}

4.1. $S_{A},-S_{C}$ Phase transition

The main goal of this investigation was a careful study of the $S_{A},-S_{C} \cdot$ phase transition of DOBAMBC. The applied electric field to the cell (figure $7(a)$ ) and the corresponding optical response near this transition and in the fully developed $\mathrm{S}_{\mathrm{C}^{*}}$. phase are represented in figure 7.

As expected the non-linear behaviour of the $\mathrm{S}_{\mathrm{C}}$. phase (figure $7(e)$ ) and the strongly increased electrooptical effect at the transition point (figures $7(c),(d)$ ) are clearly demonstrated by these measurements. However, it is also surprising that the $\mathrm{S}_{\mathrm{A}}$. phase at $84^{\circ} \mathrm{C}$ (figure $7(b)$ ) exhibits a weak non-linear behaviour probably due to a pre-transitional effect. Above $85^{\circ} \mathrm{C}$, the optical response is a very weak sinusoidal function in phase with the electric field in accord with the linear electroclinic effect [6]. Additional information is available, if these results are analysed with regard to director motions in connection with the corresponding potential function. 


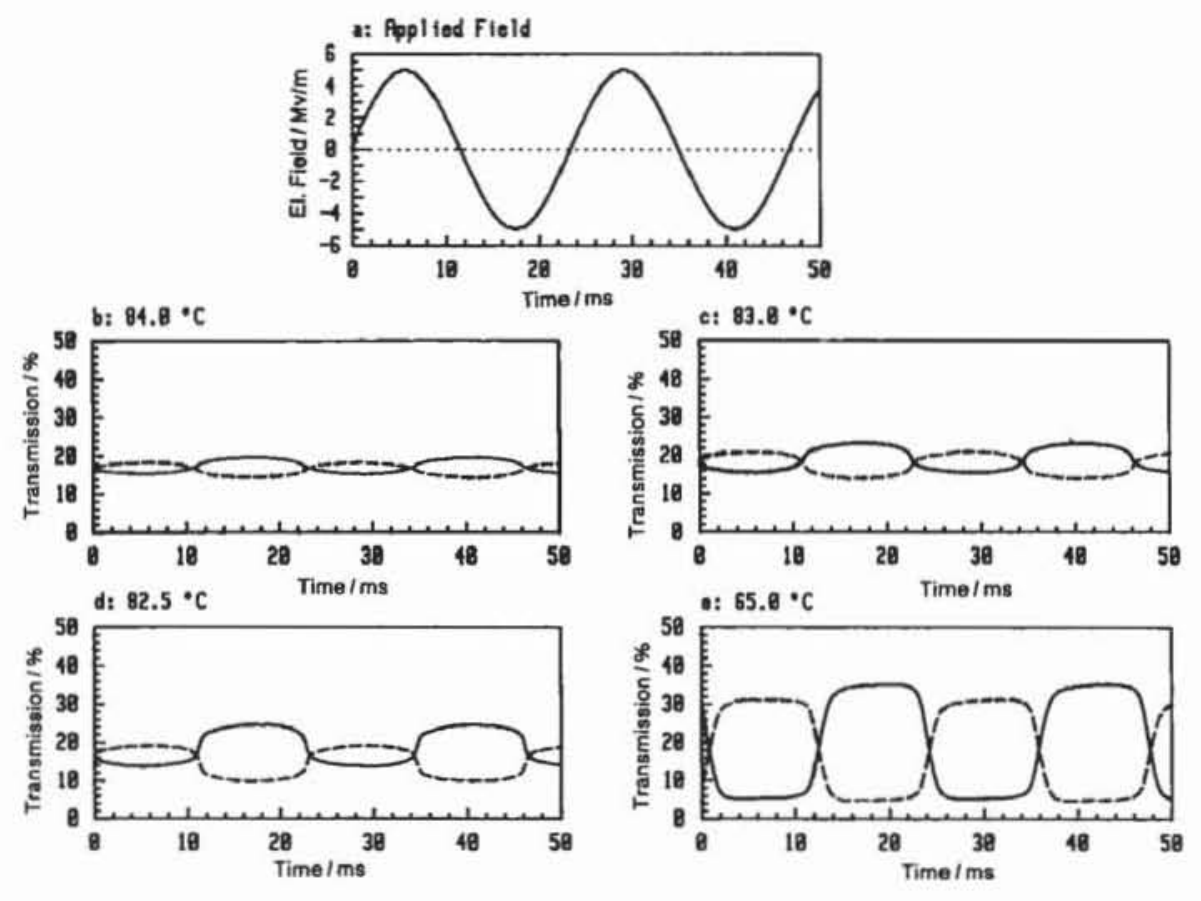

Figure 7. Electric field applied to the cell $(a)$ and optical response between crossed polars (b)-(c) at various temperatures. The optical response after a $45^{\circ}$ rotation of the polars are represented by dashed lines.

\subsection{Director movement}

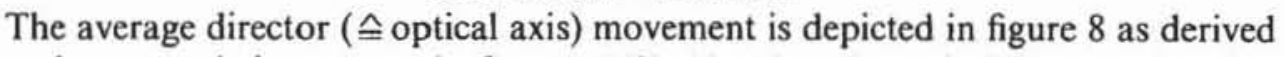
from the transmission curves in figures $7(b)-(e)$ using the uniaxial approximation according to equations (9)-(11). The ordinary refraction index $n_{\mathrm{o}}$ was determined by refractometry, and the birefringence $\Delta n=n_{\mathrm{e}}-n_{\mathrm{o}}$ of a well aligned sample measured with a Berek compensator. The results $n_{\mathrm{o}}=1.48$ and $n_{\mathrm{e}}=1.74$ agree well with published data [7]. The refractive indices and their temperature dependence do not strongly influence the calculations.

The preferred state caused by the alignment at the surface is located at negative $\varphi$ angles in all plots. All $\vartheta$ values show an average offset of about $8^{\circ}$ which is subtracted from the final values plotted in the figures. A slight layer tilt of $8^{\circ}$ may be assumed from a perpendicular orientation.

The director movement in the $\mathrm{S}_{\mathrm{A}}$. phase is described in figure $8(a)$. An almost straight line is found with only a small component in the $\vartheta$ direction at positive $\varphi$ values. Dielectric effects may be responsible for this distortion. They seem to be more restricted in the preferred surface state. The movement occurs preferentially in the $\varphi$ direction, as expected in the electroclinic effect [6].

The curves open to a slight loop near the phase transition at $83^{\circ} \mathrm{C}$ (figure $8(b)$ ). The path back to the starting point does not follow the same direction as in the $\mathrm{S}_{\mathrm{A}}$. phase at $84^{\circ} \mathrm{C}$. This trend is drastically increased only $0.5^{\circ}$ below the phase transition at $82.5^{\circ} \mathrm{C}$ shown in figure $8(\mathrm{c})$.

The fully developed motion is represented in figure $8(d)$ at a larger $\varphi, \vartheta$ scale at $65^{\circ} \mathrm{C}$. The surface stabilized state lies on the $\vartheta=0^{\circ}$ axis and the movement follows essentially along this line, with the applied electric field representing a pure $\varphi$ 

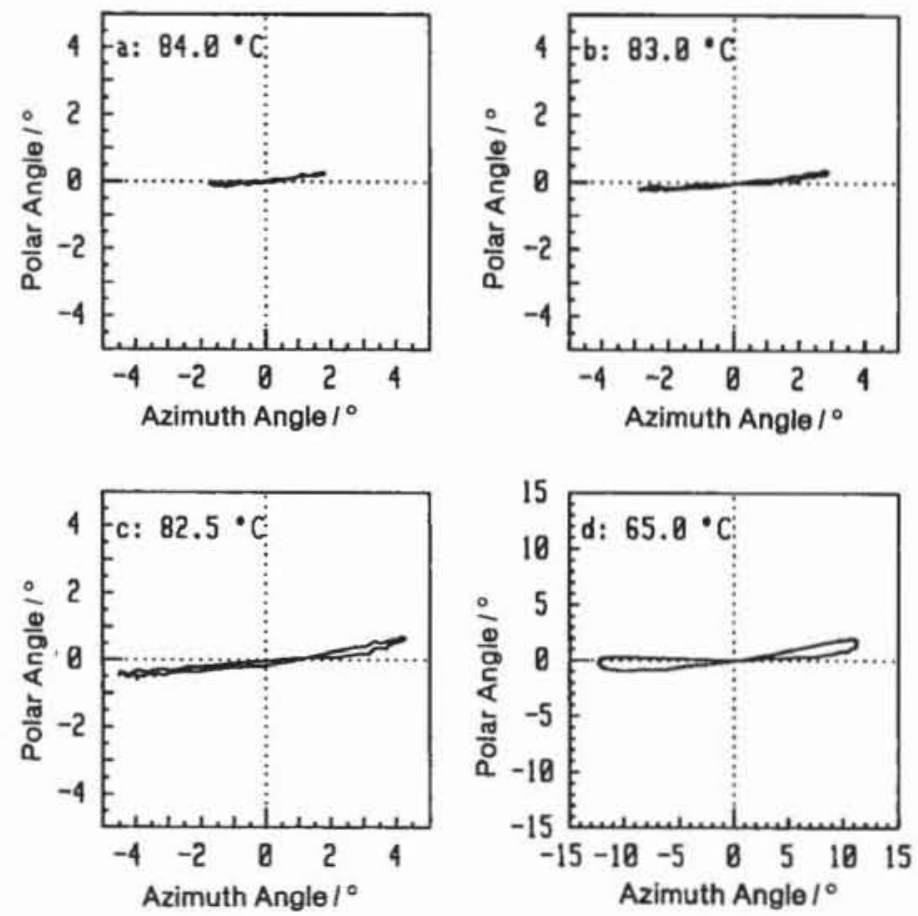

Figure 8. Average director movement of various phases at different temperatures. $\left(\varphi_{0} \approx 23^{\circ}\right.$; $n_{\mathrm{o}}=1.48, n_{\mathrm{e}}=1.74$.)

movement. The field stabilized state is placed at a small angle $\vartheta$ off the $\varphi$ axis. This measurement leads to the surprising conclusion that the average director movement does not follow the director tilt cone. The experimentally determined birefringence $\Delta n$ is constant and at its maximum value which means, that a non-uniform director field is excluded with any distribution of the director field. out of plane $|\vartheta|>0$. If the motion of the molecular level follows the average motion of the director of the smectic domains, a change of the smectic layer thickness has to occur during the switching process and/or a change in molecular conformation takes place to fit the layer thickness. The introduction of flexible parts at certain points of the molecule should then lead to faster switching times.

\subsection{Potential functions}

The hysteresis curves of figure 9 are obtained if the measured azimuthal tilt angle is plotted as a function of the applied electric field. A phase shift has to be considered between the $E$ field and the angle $\varphi$ depending on the inertia of the system. No phase correction is necessary at the relative low frequencies of about $50 \mathrm{~Hz}$ used in the measurements.

The hysteresis increases with lower temperature indicating better ferroelectric behaviour. The ferroelectric loss, repre: ented by the enclosed area, is increasing. Ferroelectric behaviour is usually stud ad by polarization-field hysteresis investigations. A prominent distinction betweer this kind of study and the proposed method is quite evident. The first derivative of the polarization with respect to time, the polarization current, is measured by the classical polarization reversal current method. The dielectric parts have to be separated and the integrated current represents the 

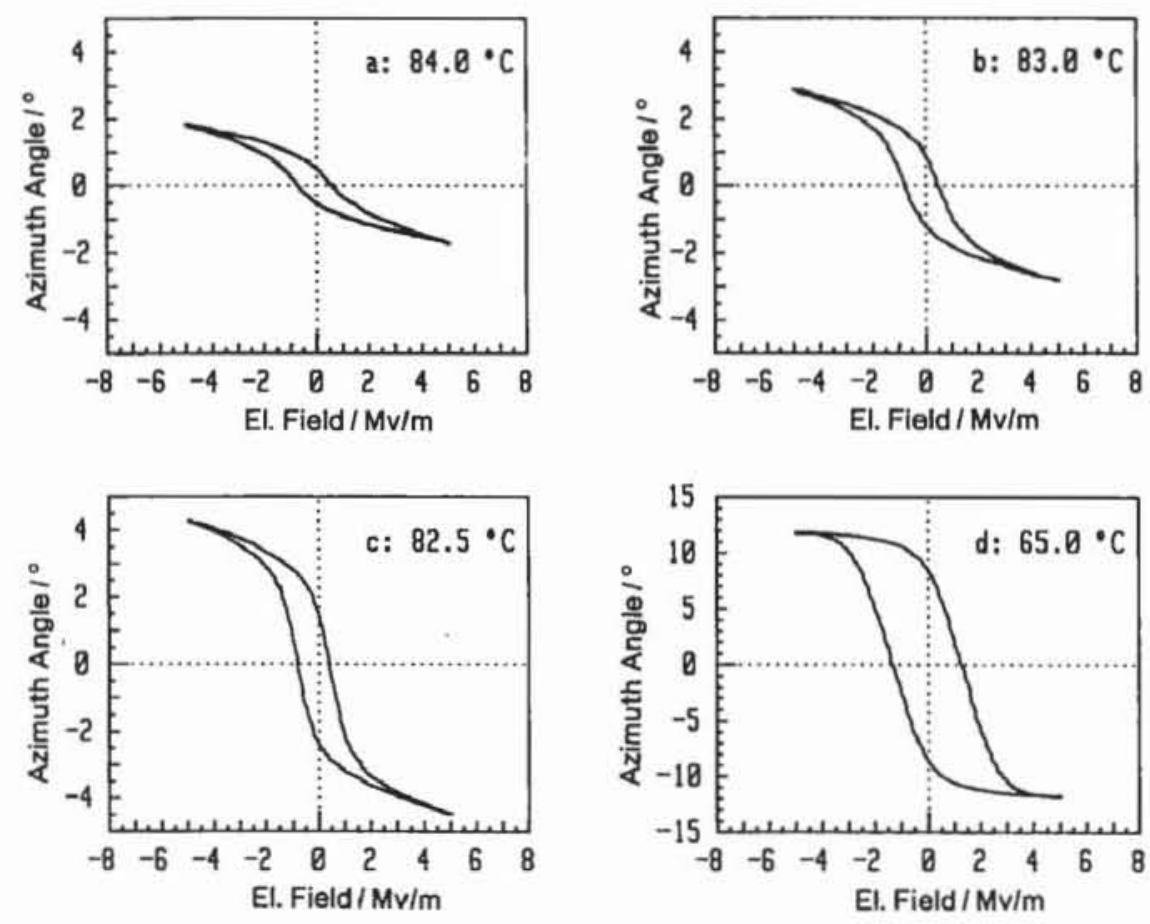

Figure 9. $E, \varphi$ hysteresis curves for various phases at different temperatures.

desired polarization. In contrast, the optical method determines the angle $\varphi$ straightforwardly, a quantity directly causing the polarization, to be applied without any integration. The difficult separation from the dielectric part is unnecessary for the material studied, since the motion caused by dipole orientation occurs essentially in the $\vartheta$ direction, the direction of the applied field.

The potential functions shown in figure 10 are obtained from the thermodynamically determined parts of the hysteresis curves and are called cell potentials. They are calculated with an arbitrary scale factor $P_{0}$ of $1 \mathrm{nC} \mathrm{cm}^{-2}$. Eliminating the orientational surface influence $\left(a_{v}=0 ; v=0,1,2, \ldots\right.$ in equation (6)), figure 11 results for the so called Landau potential. According to the Landau theory the even parts in the potential function only are necessary.

The cell and Landau potential only show one minimum and no inflexion points in the $\mathrm{S}_{\mathrm{A}}$. phase at $84^{\circ} \mathrm{C}$ (figure $10(a)$ and $11(a)$ ). The curves obviously differ from the ideal parabolic shape which result from a pure linear electric behaviour and therefore, is called a paraelectric phase. The $\mathrm{S}_{\Lambda}$. phase at $84^{\circ} \mathrm{C}$ can also be considered as a strong non-linear dielectric phase according the hysteresis curve in figure $9(a)$.

The cell-potentials show an interesting phenomena at 83.0 and $82.5^{\circ} \mathrm{C}$ (figures $10(b),(c))$. Besides a distinct minimum in the surface preferred region at negative $\varphi$ angles, two inflexion points exist. This fact can be interpreted as an ambivalent behaviour on the basis of the Landau theory [8]. The surface preferred region already exhibits real ferroelectric properties ( $\mathrm{S}_{\mathrm{C}}$. like properties) whereas in the surface discriminated region, the second ferroelectric state can only be induced $\left(\mathrm{S}_{\mathrm{A}}\right.$. like behaviour). The potential curves of figures $10(b),(c)$ represent a so-called semiferroelectric state. The appearance of intermediate semiferroelectric states leads to a broadening of the phase transition, actually observed in the polarization microscope. 

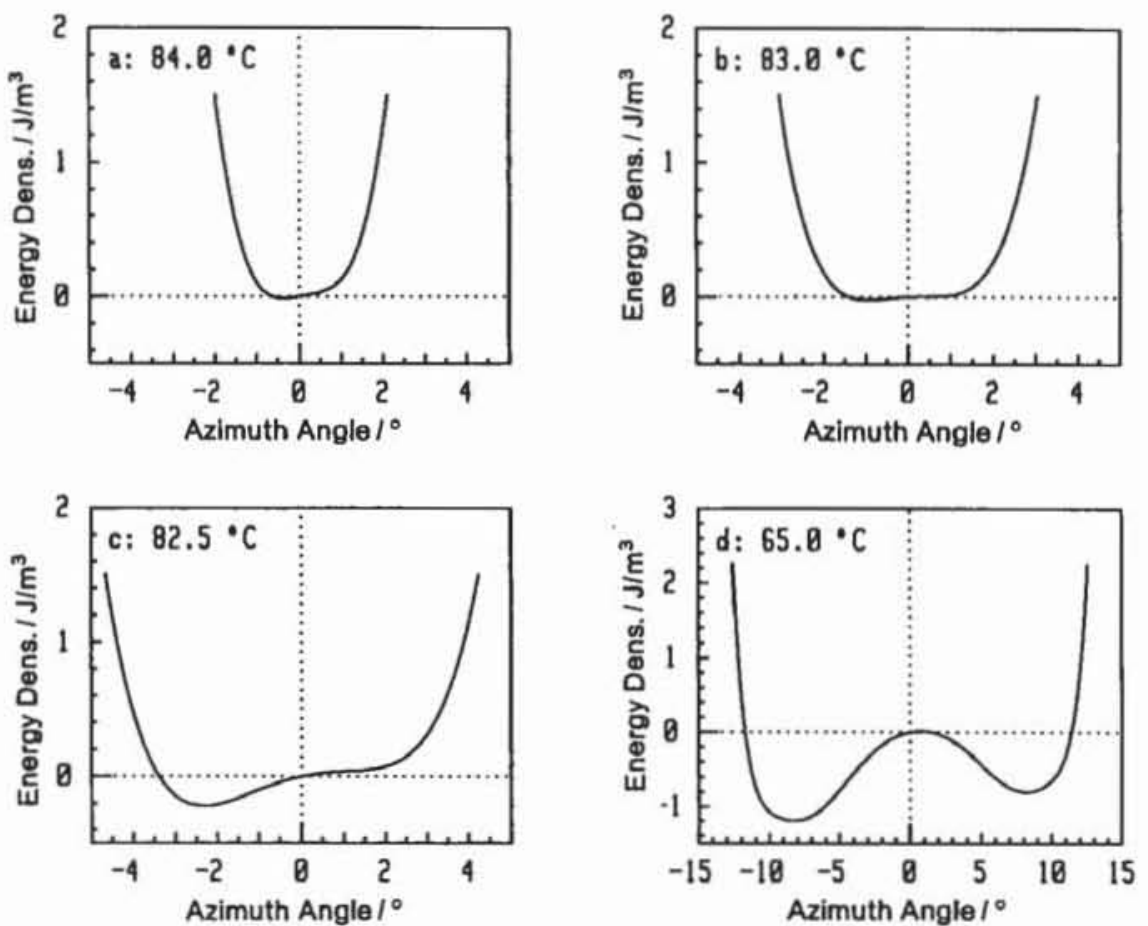

Figure 10. Cell potential curves for various phases at different temperatures calculated with $P_{0}=1 \mathrm{nCcm}^{-2}$.

In contrast, the corresponding Landau potentials show a quite normal behaviour (figure $11(b),(c)$ ). The potential curve at $83.0^{\circ} \mathrm{C}$ (figure $11(b)$ ) exhibits a broad indifferent section of about $3.5^{\circ}$ and is indicative to the situation at the immediate vicinity of the phase transition. Figure $11(c)$, taken at $82.5^{\circ} \mathrm{C}, 0.5^{\circ} \mathrm{C}$ below the temperature of figure $11(b)$, represents a typical $\mathrm{S}_{\mathrm{C}}$. potential.

The cell and Landau potential curves are shown for the completely developed $\mathrm{S}_{\mathrm{C}}$. phase in figures $10(d)$ and $11(d)$ and each exhibits two broad minima. The difference in energy between the surface preferred and surface discriminated state amounts to about $6 \mathrm{~J} \mathrm{~m}^{-3}$. The potentials of figures $10(c),(d)$ agree quantitatively with the ones calculated by Chieu et al. [5] for SSFLC cells.

\section{4. $S_{\mathrm{H}}$. phase}

Special interest has been devoted to the electrooptical behaviour to the $S_{\mathrm{H}}$. phase with new additional features as compared to the $\mathrm{S}_{\mathrm{C}}$. phase. The frequency of the applied $E$ field has to be lowered to about $7 \mathrm{~Hz}$ for the observation of the complete switching process and and the amplitude has to be increased four times more than for measurements in the $\mathrm{S}_{\mathrm{C}}$. phase.

The study was undertaken at $54^{\circ} \mathrm{C}$, one degree below the $\mathrm{S}_{\mathrm{C} \cdot}-\mathrm{S}_{\mathrm{H}}$. phase transition. The macroscopic viscosity for the higher ordered $\mathrm{S}_{\mathrm{H}}$. phase at $54^{\circ} \mathrm{C}$ is strongly increased compared to the $\mathrm{S}_{\mathrm{C}}$. phase at $55^{\circ} \mathrm{C}$. The applied $E$ field is shown in figure $12(a)$, the optical response of the system in figure $12(b)$. The switching process occurs between four differernt states as compared to two states in the $\mathrm{S}_{\mathrm{C}}$ phase. The analysis of the director movement (figure $12(c)$ ) confirms this result. Figure $12(c)$ also exhibits four stop-points at the edges of the director movement curve. Two parts are 

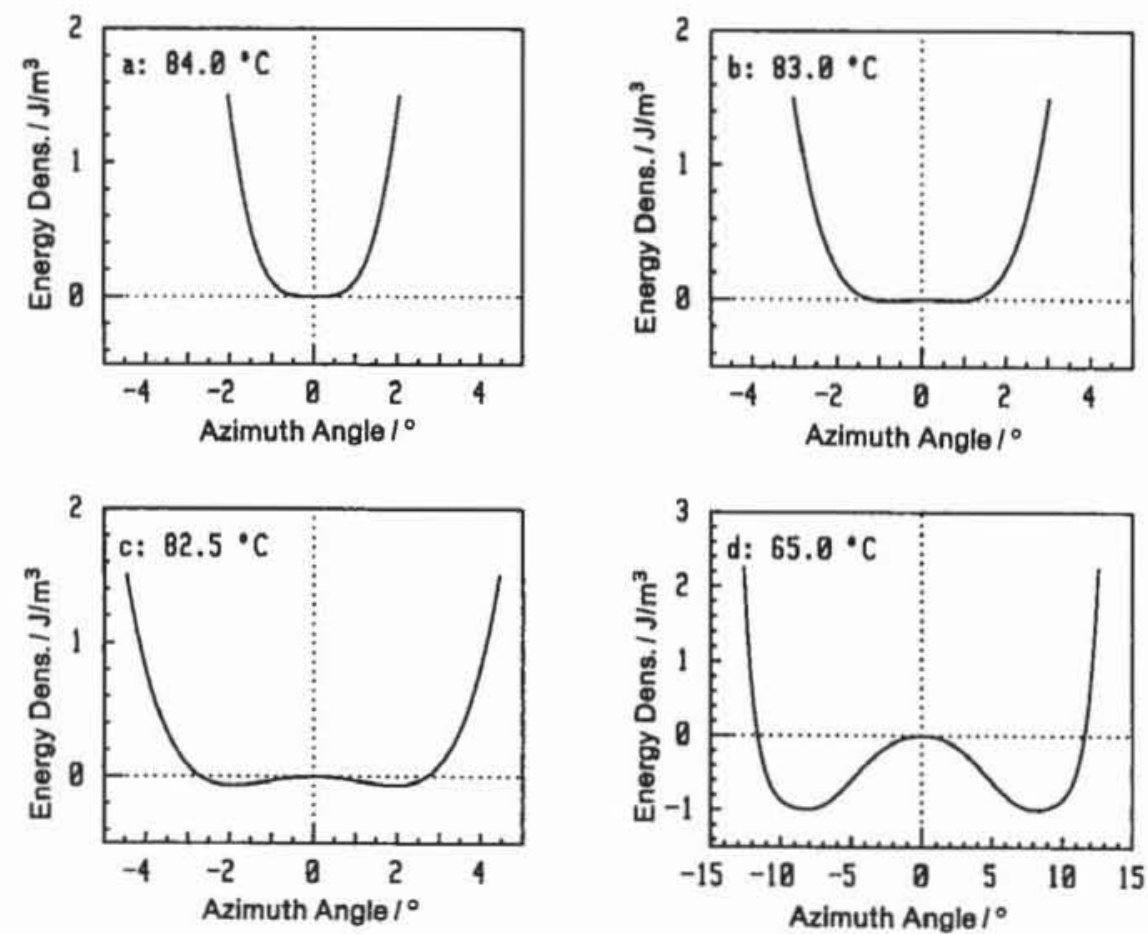

Figure 11. Landau potential curves for various phases at different temperatures calculated with $P_{0}=1 \mathrm{nC} \mathrm{cm}^{-2}$.
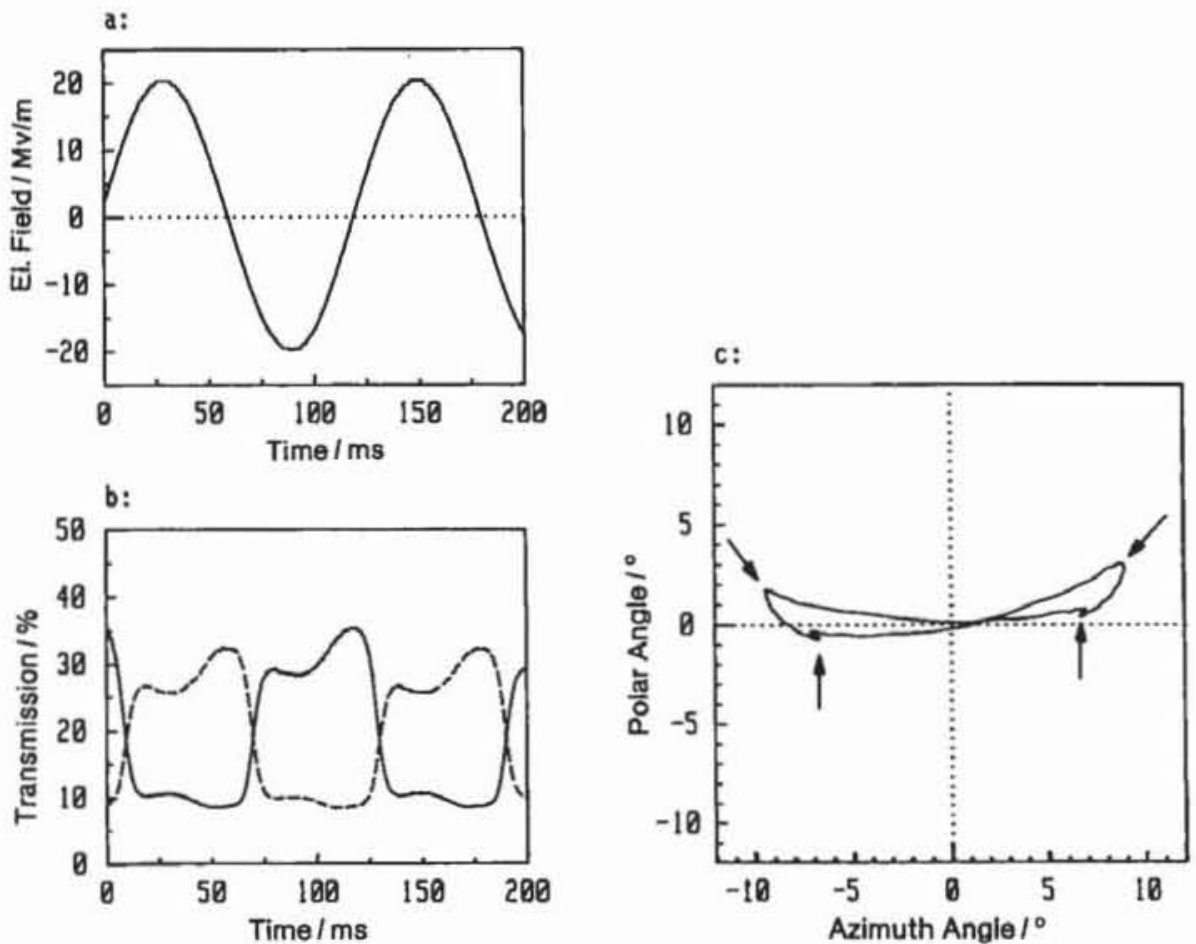

Figure 12. Electrooptical behaviour of the $\mathrm{S}_{\mathrm{H}}$. phase at $54^{\circ} \mathrm{C}$. (a) Applied electric field. (b) Optical response between crossed polars (dashed lines after a $45^{\circ}$ rotation of the polars). (c) Average director movement with $\varphi_{0}=22.8^{\circ} ; n_{\mathrm{o}}=1.48$ and $n_{\mathrm{e}}=1.74$. 
added to the $\mathrm{S}_{\mathrm{C}}$. director movement curves (cf. figures $8(a)-(d)$ ) with a preferred movement in the $\theta$ direction.

This more complex behaviour in the $\mathrm{S}_{\mathrm{H}}$. phase may be explained by the higher ordered tilted pseudohexagonal packing of the molecules in the $\mathrm{S}_{\mathrm{H}}$. layers. However, a detailed explanation of the switching mechanism in higher ordered smectics needs more experimental and theoretical investigations on the basis of structural models.

\section{Conclusions}

A generalization of the results discussed requires further investigations of $\mathrm{S}_{\mathrm{C}}$. phases with higher spontaneous polarization, other ferroelectric mesophases or ferroelectric liquid crystalline polymers. Nevertheless, the shape and symmetry of the director movement and the potential functions are useful results in the discussion of electrooptical properties.

(i) A detailed analysis of electrooptical measurements provides an efficient method for a characterization of ferroelectric liquid-crystalline material besides the interesting quantity of the switching time. The results which have been obtained are the average director movement, the temperature dependent potential function and, by an evaluation of these quantities, the availability of the orientational surface influence. The study of this influence may be of prominent interest for an optimization of alignment procedures in the design of electrooptical devices.

(ii) The experimental data for the $\mathrm{S}_{\mathrm{A}} \cdot-\mathrm{S}_{\mathrm{C}}$. phase transition and for the $\mathrm{S}_{\mathrm{C}}$. phase are described quantitatively by a modified Landau theory with additional odd terms to account for asymmetric orientational surface effects.

(iii) The transition temperatures for oriented material in thin cells differ significantly from those of the same compound in the bulk and have been observed by us for a number of benzylideneaniline derivatives [9]. The surface influence also causes new phenomena as the formation of a semiferroelectric state.

(iv) The average director of the aligned sample moves on a direct way in a plane between the two preferred states during the switching procedure of a $\mathrm{S}_{\mathrm{C}}$. phase and, since the birefringence $\Delta n$ is constant at its maximum value, a non-uniform director field with any distribution of the director field out of the plane $|\vartheta|>0$ can be excluded.

(v) The switching procedure becomes more complex in the $\mathrm{S}_{\mathrm{H}}$. phase with four preferred states.

\section{References}

[1] Meyer, R. B., LiëBert, L., Strzelecki, L., and Keller, P., 1975, J. Phys. Lett., Paris, 36, L-69.

[2] MeYer, R. B., 1977, Molec. Crystal liq. Crystals, 40, 33.

[3] Keller, P., LiëBert, L., and StrzeleCkI, L., 1976, J. Phys., Paris (Coll.), 37, C3-27.

[4] XUe, JiU-Zhi, Handschy, M. A., and Clark, N. A., 1987, Ferroelectrics, 73, 305.

[5] Chieu, T. C., Yang, K. H., and Lien, A., 1988, J. appl. Phys., 64, 6654.

[6] Garoff, S., and Meyer, R. B., 1977, Phys. Rev. Lett., 38, 848.

[7] Garoff, S., 1977, Ph.D. Thesis, Harvard University Cambridge, Massachusetts.

[8] Fatuzzo, E., and Merz, W. J., 1967, Selected Topics in Solid State Physics, Vol. VII, edited by E. P. Wohlfarth (North-Holland).

[9] Gießelmann, F., and Zugenamaier, P., 1989, Liq. Crystals, 5, 1567. Henze, H., 1989, Diploma Thesis, Institute of Physical Chemistry, Technische Universität, ClausthalZellerfeld. 